\title{
Politique
}

\section{Dynamique politique et déréglementation}

\section{Richard Carter et Michel Boucher}

Numéro 11, hiver 1987

L’État privé

URI : https://id.erudit.org/iderudit/040548ar

DOI : https://doi.org/10.7202/040548ar

Aller au sommaire du numéro

Éditeur(s)

Société québécoise de science politique

ISSN

0711-608X (imprimé)

1918-6584 (numérique)

Découvrir la revue

Citer ce document

Carter, R. \& Boucher, M. (1987). Dynamique politique et déréglementation. Politique, (11), 107-125. https://doi.org/10.7202/040548ar d'utilisation que vous pouvez consulter en ligne.

https://apropos.erudit.org/fr/usagers/politique-dutilisation/ 


\title{
DYNAMIQUE POLITIQUE ET DÉRÉGLEMENTATION
}

\author{
Richard Carter et Michel Boucher \\ École nationale d'administration publique
}

\section{Introduction}

Il est indéniable que l'on assiste à un mouvement "politique» en faveur de la déréglementation de diverses activités économiques. Autant les années $60-80$ se sont caractérisées par une vague réglementaire, autant la "déréglementation» est aujourd'hui en vogue. Cependant, si on se rappelle que la plupart des actes de réglementation se réclamaient de "l'intérêt public» sinon de l'intérêt des consommateurs, si on suppose également que très peu de ces interventions réglementaires ont finalement promu l'intérêt des consommateurs mais ont plutôt favorisé différents groupes de producteurs, on doit demeurer sceptique en face du mouvement «déréglementaire» actuel.

En effet, il y a parfois un écart considérable entre les intentions officielles des gouvernements et les résultats de leurs interventions. L'expérience de la réglementation passée doit laisser le consommateur-contribuable songeur devant les discours officiels en faveur de la déréglementation. Certes, plusieurs secteurs actuellement réglementés pourraient être soumis à une réforme dont bénéficieraient les consommateurs. Mais l'expérience observée dans plusieurs industries laisse présumer que la déréglementation ne serait 
pas nécessairement recherchée en vue de faire bénéficier les consommateurs.

Si la déréglementation est recherchée, c'est peut-être parce que la réglementation est devenue désuette pour les producteurs bénéficiaires, que de nouveaux concurrents s'insurgent contre les bénéfices qu'elle procure aux producteurs en place ainsi privilégiés ou encore parce qu'elle occasionne des coûts financiers élevés à des groupes qui détiennent désormais un certain pouvoir politique. La déréglementation peut donc surgir des mêmes circonstances que la réglementation, elle provient d'une préoccupation politique pour les intérêts de petits groupes bien organisés et non d'un quelconque attachement aux intérêts de la masse des consommateurs et contribuables. Il n'existe donc pas de théorie particulière de la déréglementation, mais seulement une théorie générale de l'action gouvernementale.

Dans cette optique, la déréglementation ne favorisera les consommateurs que si l'intérêt de différents groupes de pression les conduit à promouvoir des changements qui favoriseront également les consommateurs. Il y a peu de chances que la déréglementation soit recherchée pour le seul bien-être des consommateurs car ces derniers ne constituent pas un groupe de pression particulièrement efficace. Néanmoins, certaines réformes réglementaires peuvent contribuer au bien-être des consommateurs même si les groupes de pression qui sont à l'origine de ces réformes poursuivent leur propre intérêt économique et politique et non l'intérêt des consommateurs. Autrement dit, ce n'est que par accident que la déréglementation se réalise dans l'intérêt des consommateurs ${ }^{1}$.

Cet essai discute les raisons possibles du phénomène "déréglementaire» et souligne l'importance des groupes de pression

1. G. J. Stigler, The Citizen and the State, The University of Chicago Press, Chicago, 1975, p. 187 . "Occasionally the consumer will be protected in the legislature by another industry which happens to share the consumer's goal, but not his impotence". 
comme facteur explicatif de la déréglementation tant aux EtatsUnis qu'au Canada et au Québec.

\section{L'intérêt des groupes et la déréglementation}

Plusieurs réformes et projets de réforme sont présumément destinés à réduire le «fardeau réglementaire», à «promouvoir la productivité et la concurrence», à "protéger les consommateurs» et à assurer le «développement» économique. Dans peu de temps, ces termes deviendront naturellement les lieux communs de différents groupes de pression «de droite» tout comme la «stabilité», l' «exploitation des travailleurs» et les «droits collectifs» ont servi et servent de clichés aux groupes de pression «de gauche». Beaucoup d'entrepreneurs politiques se disent maintenant néo-libéraux, eux qui étaient sociaux-démocrates, il n'y a pas si longtemps. Les modes et les idées changent, mais la dynamique politique demeure irrémédiablement soumise au décompte des votes.

Pourquoi un gouvernement formé de politiciens rationnels préconiserait-il une déréglementation qui procurerait des bénéfices dispersés à un grand nombre d'individus, relativement amorphes dans l'arène politique, alors que les coûts d'une telle intervention seraient concentrés sur des groupes restreints, fortement organisés et enclins à exercer des pressions politiques? Peut-on parler de courage politique? D'une meilleure information des contribuables à l'égard des conséquences néfastes de la réglementation? Ou ne s'agit-il encore une fois que de l'intérêt de quelques groupes de pression qui sont avantagés par la déréglementation?

\section{Le courage politique et le cycle électoral}

On pourrait évidemment croire que le seul courage politique des hommes politiques, nouvellement élus, expliquerait cette volonté de restreindre l'emprise réglementaire de l'activité gouvernementale. Autrement dit, après avoir réalité qu'une véritable 
déréglementation est dans l'intérêt de la collectivité, le gouvernement serait disposé à l'implanter au risque de perdre plus de votes qu'il n'en gagnerait.

Cependant, on peut fortement douter que le courage politique soit en cause dans une première année de mandat électoral. Si le gouvernement du Québec maintenait sa position actuelle sur la déréglementation (rapport Scowen), la privatisation (rapport Fortier) et l'administration gouvernementale (rapport Gobeil) une ou deux années avant les élections, on pourrait fort probablement parler de courage politique. Mais trois ou quatre ans avant le déclenchement d'une élection générale, le gouvernement a suffisamment de temps pour retrouver les faveurs de l'électorat et peut se permettre quelques «bévues» ou «maladresses» politiques même si elles conduisent à une amélioration du bien-être collectif.

En d'autres termes, la période au sein du cycle politique peut constituer une variable importante pour la prise de décisions impopulaires par un gouvernement ${ }^{2}$. La probabilité de réélection, basée sur l'évaluation de sa performance par les électeurs, s'en trouve généralement peu affectée en ce que ces derniers ne retiendraient, en raison de leur myopie, que les événements les plus récents. Si on juge la façon dont le gouvernement actuel a fait face aux diverses réactions publiques, lors de la publication du rapport sur la déréglementation, on ne peut certes pas présager qu'il agira avec diligence dans un avenir immédiat. Auquel cas, la période électorale se rapprochant inéluctablement, il ne pourra plus agir sauf, évidemment, s'il possède des tendances politiques suicidaires. En bref, si le gouvernement québécois n'agit pas rapidement, il se retrouvera devant les mêmes difficultés que le

2. L'impact du cycle électoral sur les décisions économiques des gouvernements fait l'objet d'une littérature considérable. Le lecteur intéressé peut consulter B. F. S. Frey, «Politico-Economic Models and Cycles», Journal of Public Economics, 9, avril 1978, pp. 203220. 
gouvernement conservateur fédéral et ne pourra plus amorcer de réformes majeures. L'engouement du gouverneur actuel pour la déréglementation n'est peut-être donc que temporaire et éphémère en ce qu'il se situe en début de mandat.

\section{L'information économique}

Une deuxième explication possible de cette volte-face du gouvernement actuel pour la déréglementation serait le haut niveau d'éducation économique atteint par la population québécoise. Comme la masse des individus-contribuables serait devenue moins ignorante des coûts imposés par la réglementation, elle serait en mesure de mieux résister aux pressions indues des groupes restreints.

Cependant, on peut difficilement croire que la divulgation et l'explication des coûts purement financiers d'une politique gouvernementale soient suffisantes pour créer une contestation politique qui contrerait éventuellement les pressions des groupes bénéficiaires. En effet, l'analyse tant théorique qu'empirique, indique que les individus investissent davantage à acquérir de l'information sur le processus politique comme producteurs et non comme consommateurs. Par ailleurs, les coûts de la réglementation sont relativement faibles pour les consommateurs lorsqu'on les considère sur une base individuelle. Même si le coût d'une réglementation représente pour le consommateur quelques dollars de plus par chemise, paire de chaussures, poulet, litre de lait, etc., aucun consommateur ne votera, ni se prononcera contre de telles politiques qui sont officiellement destinées à «stabiliser les prix» ou à «assurer un revenu décent aux producteurs». Les sondages récents sont explicites à cet égard; ainsi, $68 \%$ des personnes interrogées lors d'un récent sondage, se sont déclarées en faveur du maintien et de l'accroissement de la réglementation actuelle (CROP, Le Devoir, 12 septembre 1986). Or pour la société dans son ensemble, les faibles coûts individuels supportés par les consommateurs, lorsque 
multipliés par le nombre de consommateurs, excèdent les bénéfices gagnés par quelques producteurs ${ }^{3}$ !

En résumé, l'éducation économique en matière de politique gouvernementale ne peut pas constituer un facteur déterminant de l'emballement du gouvernement en faveur de la déréglementation. D'autre part, en l'absence d'une sensibilisation de la population aux bienfaits de la liberté de choisir, qui ne peut être que la résultante d'un lent et long processus de formation et d'information, la dynamique politique favorisant les groupes mieux organisés s'avèrera toujours difficile à contrecarrer. Il existe cependant d'autres forces qui s'opposent naturellement aux excès réglementaires dans un monde caractérisé par un fort niveau d'interdépendance économique. Ces forces sont essentiellement la résultante d'une croissance des coûts économiques de la réglementation qui se traduit en coûts politiques.

\section{La croissance des coûts économiques de la réglementation}

La réglementation est un «service» gouvernemental dont la production par les législatures et les bureaucraties demeure soumise à une analyse économique élémentaire. Plus ce service devient coûteux en termes politiques pour les politiciens et bureaucrates, moins ces derniers sont disposés à en accroître la production. On a mentionné précédemment que les coûts politiques de la régle-

3. Par exemple, une étude de l'agence de mise ne marché des œufs de la Colombie-Britannique révèle que les coûts encourus par les consommateurs étaient de $7 \$$ par famille et que le fermier moyen recevait une subvention nette de $14000 \$$. Au total 227 propriétaires de ferme obtenaient cette rente en 1975. T. Borcherding et G. W. Dorosh, The Egg marketing Board: A Case Study of Monopoly and its Social Costs, The Fraser Institute, 1981. Une autre étude portant sur la politique protectionniste canadienne des textiles révèle que les coûts supportés par la famille moyenne sont de $101 \$$ en 79 , c'està-dire environ $33000 \$$ par emploi protégé. Cette politique a créé environ 13500 emplois supplémentaires à un salaire moyen de $10000 \$, \mathrm{G}$. P. Jenkins, "Costs and Consequences of The New Protectionism: The Case of Canada's Clothing Sector" dans Canada in a Developing World Economy: Trade or Protection, Oxford University Press, Toronto, 1983. 
mentation pouvaient croître si la population prenait davantage conscience des coûts qu'elle supporte. Néanmoins, les coûts politiques de la réglementation peuvent augmenter même si la population demeure rationnellement ignorante. En effet, ces coûts politiques peuvent s'élever si la réglementation entraîne des coûts économiques croissants à différents groupes capables d'exercer une influence politique ou si le gouvernement lui-même souffre financièrement des conséquences de la réglementation. Nous distinguons donc entre les coûts économiques et financiers imposés par la réglementation au gouvernement des coûts supportés par différents groupes d'intérêt.

\section{Les cô̂ts financiers de la réglementation pour le gouvernement}

La réglementation des activités économiques est souvent présentée comme un mode d'intervention qui est particulièrement attrayant pour les législatures. Premièrement, considérons le mécanisme des subventions croisées qui est parfois imbriqué dans la réglementation de certaines activités, comme les appels interurbains qui subventionnent les appels locaux dans le cas de la téléphonie, les parcours à grande fréquentation qui subventionnent les destinations moins achalandées dans le cas du transport aérien et par autobus, etc.. Pour obtenir des résultats équivalents, le gouvernement devrait prélever des taxes et impôts plus élevés afin de subventionner ces groupes particuliers de consommateurs. Dans cette perspective, la réglementation est politiquement intéressante en ce qu'elle permet à un gouvernement d'atteindre ses objectifs, sans modifier son budget. Autrement dit, un gouvernement procure des bénéfices à des groupes d'agents économiques par une intervention qui s'avère moins visible que s'il utilisait la taxation et les subventions directes qui gonflent le budget. Cette approche réglementaire s'avère donc particulièrement attrayante en période de restriction budgétaire. 
Deuxièmement, toujours sur le plan politique, le gouvernement peut aussi réduire ses coûts décisionnels par la réglementation. La plupart des projets de loi contiennent maintenant des clauses diverses habilitant ministres et fonctionnaires à prendre un grand nombre de décisions réglementaires. On évite ainsi à l'Assemblée Nationale de légiférer sur diverses modalités des projets de loi. En d'autres termes, la loi indique que le gouvernement est sensible à différentes situations ou problèmes politiques sans qu'aucune réflexion approfondie sur les implications de la loi ne lui soit exigée. On laisse généralement aux ministres concernés le soin de parfaire la loi au mieux de leurs connaissances, si ce n'est pas au gré des pressions politiques qui seront éventuellement exercées lors de la mise en œuvre de la loi.

Du point de vue des coûts administratifs et financiers, la réglementation possède donc des attraits indéniables pour les gouvernements. On néglige cependant qu'une trop grande abondance de règlements peut également devenir très coûteuse. Considérons, les coûts imposés par un enchevêtrement de règlements, parfois contradictoires, émis par différents ministères et organismes. À titre d'illustration la réglementation de l'environnement vise à réduire les coûts de pollution imposés par les activités de certaines industries alors que d'autres règlements, de nature strictement fiscale, peuvent encourager le développement de ces mêmes industries polluantes. La lutte à la pollution pourrait être obtenue par une taxation précise des activités polluantes (gain fiscal) alors que le gouvernement encourt dans le contexte actuel un manque à gagner fiscal ainsi que des coûts administratifs d'inspection et de contrôle. L'activité réglementaire peut donc s'avérer très coûteuse pour le gouvernement en termes non seulement d'une hausse de ses propres coûts d'opération, mais aussi d'un manque à gagner fiscal qui peut lui être associée.

On néglise souvent l'importance du manque à gagner fiscal et des dépenses que peuvent causer les multiples réglementations. 
Certes, on peut facilement saisir les avantages conférés par les exemptions, les crédits d'impôt, l'amortissement accéléré, etc. Mais on doit également considérer la diminution des activités économiques (professionnelles et industrielles) que peut provoquer la réglementation elle-même. Par exemple, de nombreuses entraves institutionnelles bloquent l'ajustement du marché du travail. Elles provoquent des dépenses supplémentaires d'assurance-chômage et d'assistance sociale d'une part, mais également un manque à gagner fiscal en ce que si ces ressources productives étaient employées, elles contribueraient aux recettes de l'État ${ }^{4}$. Toute réglementation des marchés qui empêche l'expansion économique et par conséquent l'emploi implique un manque à gagner fiscal au gouvernement. Ainsi plus la réglementation entrave le libre fonctionnement des marchés, plus elle devient dispendieuse pour le gouvernement en termes du manque à gagner fiscal occasionné.

Si on suppose qu'un des objectifs du gouvernement est d'accroître ses revenus ${ }^{5}$, on comprend que l'intervention «réglementaire» finit par coûter de plus en plus cher en termes des revenus fiscaux qui ne peuvent plus être puisés dans l'économie

Finalement, il est utile de rappeler que la cote de crédit du gouvernement du Québec s'est améliorée sur le marché nordaméricain immédiatement après la publication-des rapports sur la déréglementation, la privatisation et la réforme administrative. Les intentions de libéralisation des marchés qui sont énoncées dans ces rapports ont apparemment permis la réalisation immédiate de gains financiers par le gouvernement en ce qu'elles ont réduit le taux d'intérêt qu'il doit débourser pour assurer le service de la dette publique. Sans prétendre que ces rapports soient la seule

4. R. Carter, «Entraves institutionnelles et emploi au Québec», Gestion, septembre 1984, pp. 27-33.

5. Pour une analyse constitutionnelle des conséquences de cet objectif, il faut consulter J. M. Buchanan \& G. Brennan, The Power to Tax, Cambridge, University Press, 1980. 
cause de l'amélioration de la cote de crédit du gouvernement, il n'en demeure pas moins que sa position, favorable à l'égard de l'expansion économique, n'a certainement pas nui à l'évaluation de sa situation financière propre.

Si une réglementation excessive des marchés conduit à l'anticipation, chez les bailleurs de fonds éventuels, que l'économie nationale ne pourra croître qu'à un rythme ralenti, il est alors vraisemblable qu'on anticipe aussi que le gouvernement aura de plus grandes difficultés à prélever les taxes requises au remboursement de ses dettes. La cote de crédit du gouvernement est alors diminuée et ce dernier est confronté à des coûts financiers immédiats qui sont plus élevés. La réglementation des marchés économiques par les gouvernements peut leur coûter des frais de financement plus élevés. De plus, si l'endettement gouvernemental est déjà considérable, une faible hausse de taux d'intérêt peut engendrer des frais supplémentaires importants.

Ces quelques considérations financières permettent de comprendre que si le gouvernement est dans une situation difficile, il peut lui-même chercher à réduire le fardeau réglementaire pour des raisons purement financières. Il y a cependant d'autres forces qui peuvent accélérer le mouvement de la déréglementation.

\section{Les coûts de la réglementation pour les industries}

Nous avons souligné que certaines formes de réglementation favorisent différents groupes d'intérêts, qu'il s'agisse de groupes d'entreprises, d'industries ou de travailleurs. Évidemment, lorsqu'un groupe est favorisé, un autre est généralement défavorisé. Dans plusieurs cas, les groupes défavorisés sont les masses de consommateurs et de contribuables. Dans certains autres cas, on retrouve également des groupes industriels qui peuvent accéder, éventuellement, à un pouvoir politique suffisamment grand pour contrer les pressions exercées par les groupes bénéficiaires. Finalement, 
il y a aussi les groupes pour lesquels les bénéfices de la réglementation se sont graduellement estompés et pour qui la déréglementation devient bénéfique.

Plusieurs exemples viennent étayer cette approche à la déréglementation. Ainsi, un des principaux facteurs permettant d'expliquer la déréglementation du transport ferroviaire, des télécommunications et de l'aviation commerciale aux États-Unis se trouve dans la désuétude de la réglementation même. En particulier, les systèmes de subventions croisées se sont éventuellement écroulés dans chacune de ces industries. Plusieurs mécanismes expliquent l'érosion graduelle de la réglementation pour les groupes qui l'ont recherchée.

Dans le cas de l'aviation américaine, T. E. Keeler résume l'histoire de la déréglementation de la façon suivante:

"Airline regulation was not working because it had set out to cross-subsidize lowdensity routes with profits from high-density routes. But, as many students of airline regulation have argued, service quality competition on high density routes tended to eliminate excess profits on these routes, and airlines not receiving adequate subsidies to operate low-density routes withdrew services from those routes" ${ }^{6}$.

En d'autres termes, la réglementation des tarifs aériens permettait des profits de cartel sur les routes achalandées. Mais comme on ne pouvait pas bloquer la concurrence par la qualité des services, ces profits se sont dissipés sous la forme de coûts d'opération plus élevés. Les profits des routes achalandées ne pouvaient donc plus être utilisés pour subventionner les routes moins achalandées. Plusieurs compagnies en place sont alors devenues favorables à un retour à la concurrence par les prix que permettrait la déréglementation. Notons, par ailleurs, que l'apparition de transporteurs aériens non-réglementés à l'échelle internationale (Lakers Airlines) avait permis à la population de découvrir l'im-

6. T. E. Keeler, "Theories of Regulation and the Deregulation Movement ", Public Choice, vol. 44, no 1, 1984, p. 113. 
portance des économies qu'elle pouvait réaliser par la déréglementation et que des pressions politiques s'étaient exercées à cet effet.

Une situation relativement similaire s'est produite pour la déréglementation du transport ferroviaire aux États-Unis. Cette fois cependant, la capacité de fixer des prix anormalement élevés pour le transport de certaines marchandises sur des trajets spécifiques, présumément dans le but de subventionner le transport d'autres marchandises sur d'autres trajets, fut graduellement éliminée par la concurrence du camionnage:

\begin{abstract}
«... competition, especially from highways, eroded the railroad's most profitable business, and made the unprofitable business an even greater drain on railroad finances. As a result, regulation simply could not generate enough revenue in the railroad industry to cross-subsidize all the services deemed politically necessary and desirable by Congress and the regulators»?
\end{abstract}

Dans le cas des télécommunications, ce sont les nouvelles technologies qui ont permis l'apparition de concurrents pour les services téléphoniques interurbains. Le monopole d'AT\&T ne pouvant alors être maintenu face aux pressions de nouveaux concurrents, il était devenu impossible de subventionner les appels locaux à l'aide d'une tarification monopolistique des appels interurbains.

En somme, dans ces trois cas de déréglementation américaine, la réglementation était devenue "coûteuse» pour les industries initialement bénéficiaires. Des facteurs de dissipation de la rente aussi variés que la concurrence par la qualité pour l'aviation commerciale, l'existence de substituts pour le chemin de fer et la nouvelle technologie pour les télécommunications en sont directement responsables. Ces industries avaient donc de moins en moins d'intérêt à s'objecter à la déréglementation. On peut même croire que la «déréglementation» a permis de réduire les pertes

7. T. E. Keeler, op. cit., p. 109. 
encourues par certaines entreprises qui étaient encore obligées de fournir des services à des prix inférieurs aux coûts de production.

En effet, comme les forces de la concurrence ont graduellement éliminé les profits des activités rentables qui finançaient les activités non-rentables, les entreprises devaient alors supporter les pertes occasionnées par ces dernières. Selon Keeler, c'est désormais le gouvernement américain, la masse non organisée des contribuables, qui en assume maintenant les frais en ce qu'il les subventionne:

«Furthermore, for both rail and air service, direct government subsidies are being used to support unprofitable services in lieu of cross-subsidies. And finally, in telecommunications, a special fund is being set up as a way of continuing crosssubsidies which would otherwise be eliminated"8.

On peut donc croire qu'une part importante du mouvement américain de la déréglementation est le résultat d'une croissance des pertes encourues par les industries réglementées. La concurrence sous toutes ses formes s'est finalement infiltrée au sein du processus réglementaire et l'a fait éclaté.

Des facteurs similaires de concurrence interne ainsi que l'ouverture de l'économie canadienne à la concurrence étrangère soustendent et expliquent actuellement le phénomène canadien (et québécois) de déréglementation partielle des industries du transport ${ }^{9}$. Des firmes américaines de chemin de fer et de camionnage exercent des pressions constantes sur la structure réglementaire canadienne (provinciale et fédérale) en opérant en territoire canadien. Elles sont alors en mesure d'offrir leurs services à des prix inférieurs à ceux des entreprises canadiennes réglementées. La concurrence des compagnies aériennes déréglementées expose aussi Air Canada

8. T. E. Keeler, op. cit., p. 120.

9. Le même phénomène se retrouve en télécommunications où la possibilité de réaliser des subventions croisées dans le secteur de la téléphonie est compromise par les pressions exercées sur le CRTC par des compagnies qui veulent offrir de nouveaux services interurbains. Il faut consulter $R$. Carter, "Le Canada est-il entrainé dans la déréglementation: le cas des communications", Analyse de politiques, X, 1, mars 1984, pp. 10-24. 
à encourir des déficits sur les trajets intercontinentaux pour maintenir sa part de marché alors que l'expérience américaine de prix réduits a rendu plus visibles les pratiques monopolistiques de cette entreprise publique pour les trajets nationaux ${ }^{10}$.

Au Canada tout comme au Québec, le mouvement de déréglementation est donc partiellement motivé par la déréglementation américaine qui accroît les coûts politiques et économiques de toute réglementation rendant les industries moins concurrentielles sur le plan international. À cet égard, les industries nationales ne pourront s'opposer longtemps à la déréglementation car elles perdront graduellement leurs ventes au profit des compagnies américaines. Bientôt, elles percevront leur propre intérêt dans un retour à la concurrence ${ }^{11}$.

Différents extraits du rapport Scowen sur la déréglementation ${ }^{12}$ indiquent également que ce dernier fut influencé par les groupes de pression. Plus exactement, ce rapport ne recommande aucune mesure libéralisatrice qui pourrait introduire la concurrence sur le marché du travail. Et la recommandation d'abolir les décrets d'extension de convention collective semble avoir été influencée par les représentants de certaines entreprises exportatrices, principalement le meuble et le vêtement. Par ailleurs dans son analyse des quelques thèmes traités, le groupe adhère aux politiques des groupes organisés et structurés. En matière de transport routier des marchandises, il recommande la déréglementation presque totale parce que les échanges canado-américains et la présence de

10. M. Boucher, «La dérégulation américaine des transports», L'Analyste, 10, été 1985, pp. 50-53.

11. Pour une appréciation des conséquences intrinsèques de la réglementation américaine et de sa propagation en territoire québécois, il faut consulter $\mathbf{M}$. Boucher, "L'inspiration américaine de la déréglementation en transport routier", Analyse de politiques, XII, mars 1986, pp. 189-201.

12. Groupe de travail sur la déréglementation, Réglementer moins et mieux, Les publications du Québec, juin 1986. 
transporteurs américains en territoire québécois en favorisent la diffusion de ce côté-ci du $45^{\text {ième }}$ parallèle.

Dans plusieurs cas on peut conclure que les consommateurs bénéficieront de la déréglementation même si elle est mise en place à la suite de pressions d'entreprises. En effet, les consommateurs voient souvent leurs intérêts mieux protégés par les forces d'un marché concurrentiel que par un marché qui est cartellisé par l'intervention réglementaire de l'État. De nouvelles entreprises ont toujours intérêt à pénétrer un cartel afin de bénéficier des profits anormalement élevés qu'il confere. Mais leur entrée dans un cartel signifie également son éclatement éventuel et l'apparition de prix concurrentiels inférieurs. Que ce soit pour bénéficier des profits d'un cartel ou encore pour accroître leurs parts relatives de marché, les firmes domestiques et étrangères peuvent donc servir l'intérêt des consommateurs lorsqu'elles tentent de détruire les règlements qui nuisent à la concurrence. Dans cette perspective restreinte, les entreprises qui «financent» les pressions politiques ou qui font de l'action politique pour la déréglementation d'une activité permettent ultimement l'avancement de l'intérêt des consommateurs.

Mais cette dynamique de la déréglementation peut également jouer au détriment des consommateurs et des contribubles dans plusieurs cas. Le terme déréglementation devient alors un fourretout où on risque de trouver un grand nombre d'interventions qui favorisent exclusivement les producteurs et non les consommateurs. Ce danger de la déréglementation fut noté dans les termes suivants par D. Needham:

"If interest groups that desire increased regulation perceive that their demands will be more effective if couched in terms of deregulation, the terminology used by various groups involved in the legislative process may be an unreliable guide to the actual content of legislation that embodies regulatory features " 1 .

13. D. Needham, The Economics and Politics of Regulation: A Behavioral Approaich, Little, Brown \& Company, Boston, 1983, p. 399. 
Ainsi, par exemple, l'étude spécifique des compensations versées aux chemins de fer lors de la réforme de l'Accord du Nid de Corbeau (1983) laisse croire que cette «déréglementation" fut complètement contrôlée par deux groupes de pression particulièrement efficaces, les agriculteurs et les compagnies ferroviaires. Le résultat de la réforme fut essentiellement de transférer aux contribuables canadiens le déficit annuel des compagnies ferroviaires $^{14}$.

Que dire par ailleurs des recommandations d'un groupe de travail sur la déréglementation dont le mandat consiste à «formuler des recommandations destinées à placer les entreprises du Québec dans une situation au moins aussi avantageuse que celles de l'Ontario» ${ }^{15}$. On ne peut alors être surpris de l'absence de recommandations sur le précompte syndical (formule Rand) "parce que son existence a suscité très peu d'objections de la part des entreprises... ${ }^{16}$. Néanmoins des changements de fond et de forme s'imposent dans le cas des réglementations sur la santé et la sécurité au travail car: "le régime en vigueur est, de loin, la plus grande source d'insatisfaction des dirigeants d'entreprise au Québec ${ }^{17}$.

En d'autres termes, on fait des propositions afin de réduire les coûts économiques et d'accroître la flexibilité interne des entreprises québécoises. Le Conseil du patronat semble avoir remplacé les grands syndicats comme groupe de pression déterminant, mais l'intérêt de la masse des consommateurs et contribuables demeure absent du mécanisme d'évaluation.

La même dynamique se retrouve dans les recommandations sur le salaire minimum et le code du travail. Une vision plus

14. R. Carter, "Déréglementation: faut-il compenser les groupes qui sont pénalisés", Analyse de politiques, XII, 2, juin 1986, pp. 294-306.

15. Groupe de travail sur la déréglementation, op. cit., p. 1.

16. Groupe de travail sur la déréglementation, op. cit., p. 115.

17. Groupe de travail sur la déréglementation, op. cit., p. 102. 
réaliste aurait conduit à reconnaître que certains groupes bénéficient des rigidités imposées aux travailleurs les moins qualifiés par le salaire minimum. Pensons aux travailleurs qui subissent directement leur concurrence, soit ceux jouissant de qualifications tout juste supérieures ou ceux qui reçoivent un salaire syndical supérieur au salaire concurrentiel, et aux entreprises de grandes régions industrielles qui voient les petites firmes des régions périphériques ne plus être en mesure de les concurrencer efficacement en employant des procédés de production plus laboriques (labour intensive) ${ }^{18}$.

Un autre thème traité par le rapport Scowen est celui de l'accès à l'égalité pour les femmes. Les entreprises consultées par le groupe ne se sont pas plaintes de l'article 19 de la charte des droits et libertés de la personne qui stipule que tout employeur doit, sans discrimination, accorder un salaire égal pour un travail de valeur équivalente. Par conséquent, le groupe propose le maintien du statu quo. Cela signifie probablement que la loi n'est effectivement pas contraignante pour ces entreprises et n'occasionne pas des coûts anormalement élevés ${ }^{19}$.

Doit-on rappeler que les mouvements féministes, composées des femmes les plus qualifiées, les plus visibles et les plus matériellement favorisées, représentent un lobby aussi puissant au Québec que dans les autres provinces. Le groupe de travail ne mentionne nulle part la discrimination indirecte associée aux réglementations publiques comme certaines interdictions faites aux femmes d'accéder à certains emplois, que la discrimination a été plus marquée dans les emplois publics que dans les entreprises

18. P. Linneman, «The Economic Impacts of Minimum Wage Laws: A New Look at an Old Question», Journal of Political Economy, 90, juin 1982, pp. $443-469$ et J. Silberman et G. Durden, "Determining Legislative Preferences on the Minimum Wage», Journal of Political Economy, 84, avril 1976, pp. 317-330.

19. Prenons par exemple la position de l'administration fédérale qui s'est le plus avancée en matière de discrimination positive (affirmative action). La contrainte imposée aux employeurs obtenant des contrats se limiterait aux contrats de valeur minimale et se voudrait plus incitative que coercitive. De plus une simple déclaration de bonnes intentions de l'entreprise suffirait. 
marchandes ${ }^{20}$ et que le gouvernement s'est opposé à la promotion féminine en accordant à des groupes de pression le pouvoir de coercition nécessaire à de telles pratiques discriminatoires ${ }^{21}$.

\section{Conclusion}

L'analyse de la déréglementation révèle que cette dernière fut souvent recherchée pour des motifs tout autre que la poursuite de l'intérêt des consommateurs. Les gouvernements peuvent être incités à déréglementer parce que l'activité réglementaire accroît leurs coûts d'opération, et augmente leur manque à gagner fiscal en réduisant le rythme de l'activité économique. Cependant la principale raison semble provenir des coûts élevés que la réglementation impose aux industries réglementés et qui les conduisent éventuellement à rechercher son rejet.

Nous ne croyons pas que des réformes d'envergure seront dictées et mises en application par simple souci d'accroître l'efficacité économique sinon le bien-être des consommateurs. Dans cette perspective, aucune des recommandations touchant le marché du travail que le rapport Scowen définit erronément sous le vocable de réglementation sociale, n'aura de fortes chances d'être implantée que si des groupes d'intérêt puissants, comme le Conseil du patronat, en font leur cheval de bataille.

On peut sérieusement se demander en quoi le gouvernement actuel pourra éviter de se conformer à cette réalité politico-économique qui a dicté le comportement de tant de gouvernements démocratiques? Comment le gouvernement du Québec peut-il vaincre cette dynamique politique inéluctable où le décompte des

20. A. Alchian et R. A. Kessel, "Competition, Monopoly and the Pursuit of Money» dans A. Alchian, Economic Forces at Work, Liberty Press, Indianapolis, 1977, pp. 151-176.

21. Pour une analyse de ce problème, il faut consulter J.-L. Migué, «Discrimination positive et égalité des femmes au travail", Centre de recherche en économie publique, cahier 8604, École nationale d'administration publique, 1986, p. 34. 
votes tient souvent lieu de rationalité collective, où les ministères et les technocrates qui conseillent le gouvernement ne sont parfois que les porte-paroles de différents groupes de pression?

Dans ce contexte, il n'y a que deux forces qui puissent inciter à la déréglementation. La première est essentiellement aléatoire et est constituée par la venue de nouveaux concurrents qui, comme pour les industries du transport ferroviaire et routier, de l'aviation commerciale et des télécommunications feront éventuellement éclater les réglementations protectionnistes. La déréglementation se réalise parce qu'elle ne procure plus de rentes aux facteurs concernés.

La deuxième découle de l'observation que le problème fondamental de toute démocratie est d'être facilement dominée par des groupes de pression. Seules des contraintes sur le comportement effectif des hommes politiques peuvent réduire les inefficacités inhérentes au processus politique. Autrement dit, il faut contraindre les comportements effectifs des politiciens pour qu'ils cessent de promouvoir des politiques dont les bénéfices sont concentrés à des groupes spécifiques et les coûts dispersés à l'ensemble des contribuables. Ces limites effectives au rôle d'un homme politique ne peuvent venir que de l'extérieur et doivent être incorporées dans la constitution. Il faudrait créer une Charte des droits économiques du citoyen qui empêcherait l'État d'intervenir d'une manière discriminatoire sur le territoire ${ }^{22}$. Cette proposition de changement constitutionnel découle de la constatation que le politicien, comme tout autre membre d'une société, est mû par son intérêt personnel. Il ne fera la promotion de l'intérêt des consommateurs que s'il est induit, par des contraintes constitutionnelles, à agir ainsi. Seule cette garantie assurera l'empressement du politicien à la promotion de l'intérêt des consommateurs.

22. H. G. Grubel, "A Canadian Bill of Economic Rights», Analyse de Politiques, VIII, mars 1982, pp. 57-68. 